\title{
THE GROWTH OF HYPOELLIPTIC POLYNOMIALS AND GEVREY CLASSES
}

\author{
E. NEWBERGER AND Z. ZIELEZNY
}

\begin{abstract}
For given hypoelliptic polynomials $P$ and $Q$, classes $\Gamma_{P}^{\rho}(\Omega)$ and $\Gamma_{Q}^{\rho}(\Omega)$ involving Gevrey type estimates on the successive iterates of the corresponding differential operators are defined. The equivalence of the inequality $|Q(\xi)|^{2} \leqq C\left(1+|P(\xi)|^{2}\right)^{h}, \xi \in R^{n}$, and the inclusion $\Gamma_{P}^{\rho}(\Omega) \subset \Gamma_{Q}^{\rho^{h}}(\Omega)$ is proved.
\end{abstract}

A differential polynomial $P(D)$ on $R^{n}$ of order $m$ is elliptic if and only if it satisfies the growth condition

$$
|\xi|^{2 m} \leqq C\left(1+|P(\xi)|^{2}\right), \quad \xi \in R^{n},
$$

where $C$ is a constant (see e.g. [1, p. 75]).

Another characterisation of the ellipticity of $P(D)$, based on a theorem of $\mathrm{H}$. Komatsu [3], is given by the following condition:

A $C^{\infty}$-function $u$ in a domain $\Omega \subset R^{n}$ is analytic in $\Omega$ if and only if, for every compact subset $K \subset \Omega$, it satisfies the estimates

$$
\left\|P^{j}(D) u\right\|_{K} \leqq A^{j+1}(j !)^{m}, \quad j=0,1, \cdots,
$$

where \|\|$_{K}$ is the $L^{2}$-norm over $K$ and $A$ is a constant depending on $u$ and $K$.

Conditions (I) and (II) are therefore equivalent.

In this note we prove that the above equivalence is a particular case of a more general correspondence between the growth of hypoelliptic polynomials on $R^{n}$ and the width of classes of $C^{\infty}$-functions defined by some "Gevrey type" estimates.

Let $\Omega$ be an open subset of $R^{n}$ and $\rho$ a nonnegative number. We denote by $\Gamma^{\rho}(\Omega)$ the $\rho$ th Gevrey class in $\Omega$, i.e. the set of functions $u \in C^{\infty}(\Omega)$ with the property: To every compact set $K \subset \Omega$ there exists a finite constant $A$ (depending on $u$ and $K$ ) such that

$$
\sup _{x \in K}\left|D^{\alpha} u(x)\right| \leqq A^{|\alpha|+1}\left(\alpha_{1} !\right)^{\rho}\left(\alpha_{2} !\right)^{\rho} \cdots\left(\alpha_{n} !\right)^{\rho},
$$

for any multi-index $\alpha=\left(\alpha_{1}, \alpha_{2}, \cdots, \alpha_{n}\right)$.

Received by the editors April 21, 1972.

AMS (MOS) subject classifications (1970). Primary 35H05; Secondary 35J30, 26 A93. class.

Key words and phrases. Elliptic differential operator, hypoelliptic polynomial, Gevrey

(c) American Mathematical Society 1973 
In view of Sobolev's lemma (see [3, Lemma 2]), condition (1) is equivalent to the condition

$$
\left\|D^{\alpha} u\right\|_{K} \leqq B^{|\alpha|+1}\left(\alpha_{1} !\right)^{\rho}\left(\alpha_{2} !\right)^{\rho} \cdots\left(\alpha_{n} !\right)^{\rho}
$$

with another constant $B$.

If $P$ is a polynomial on $R^{n}$, we denote by $\Gamma_{P}^{\rho}(\Omega)$ the set of all functions $u \in C^{\infty}(\Omega)$ with the property: To every compact set $K \subset \Omega$ there exists a finite constant $B$ (depending on $u, K$ and $P$ ) such that

$$
\left\|P^{j}(D) u\right\|_{K} \leqq B^{j+1}(j !)^{\rho}, \quad j=0,1, \cdots .
$$

Note that in conditions $\left(1^{\prime}\right)$ and (2) we can use the powers $\alpha_{j}^{\alpha_{j}}$ and $j^{j}$, respectively, instead of the factorials (Stirling formula!). In that case it is convenient to set $0^{\circ}=1$.

If $u$ is a function on $\Omega$ and $\Omega^{\prime} \subset \Omega$, we denote by $u \mid \Omega^{\prime}$ the restriction of $u$ to $\Omega^{\prime}$. We also write $\Omega^{\prime} \subset \subset \Omega$ to designate that the closure $\bar{\Omega}^{\prime}$ is compact and $\bar{\Omega}^{\prime} \subset \Omega$.

Let now $P$ be a hypoelliptic polynomial and $Q$ an arbitrary polynomial. Then there is an $h>0$ such that, for some constant $C$,

$$
|Q(\xi)|^{2} \leqq C\left(1+|P(\xi)|^{2}\right)^{h}, \quad \xi \in R^{n} .
$$

Moreover, there exists a smallest such $h$, which is a rational number (see [2, Theorem 3.2]).

THEOREM 1. Assume that $P$ and $Q$ are hypoelliptic polynomials satisfying condition (3) with some $h>0$. Then, for any open set $\Omega \subset R^{n}$ and large $\rho$,

$$
\Gamma_{P}^{\rho}(\Omega) \subset \Gamma_{Q}^{\rho h}(\Omega) .
$$

Proof. If the inclusion (4) holds for some $h$, it holds also for every $h^{\prime}>h$. Thus, by what we have said before, it is sufficient to prove the theorem for $h=\mu / v$, where $\mu$ and $v$ are integers $>0$. In this case condition (3) can be written in the form

$$
\left|Q^{v}(\xi)\right|^{2} \leqq C\left(1+\left|P^{\mu}(\xi)\right|^{2}\right), \quad \xi \in R^{n},
$$

with some constant $C$ (not necessarily the same as in (3)).

Let $\Omega$ be a bounded open subset of $R^{n}$ and $\Omega_{\delta}, \delta>0$, the set of points $x \in \Omega$ whose distance from $\mathrm{C} \Omega$ is greater than $\delta$. By a theorem of $\mathrm{L}$. Hörmander [2, Theorem 4.2], condition (5) implies that, for some $\gamma>0$ and every $s \geqq 0$ and $t>0$,

$$
\sup _{0<\tau \leqq t} \tau^{\gamma}\left\|Q^{v}(D) v\right\|_{\Omega_{s+r}} \leqq C\left\{\sup _{0<\tau \leqq t} \tau^{\gamma}\left\|P^{\mu}(D) v\right\|_{\Omega_{s+r}}+\|v\|_{\Omega_{s}}\right\}, \quad v \in C^{\infty}\left(\Omega_{s}\right),
$$


where $C$ is a constant depending only on $P, Q$ and the diameter of $\Omega .^{1}$ Hence

$$
\left\|Q^{v}(D) v\right\|_{\Omega_{s+t}} \leqq C\left\{\left\|P^{\mu}(D) v\right\|_{\Omega_{s}}+t^{-\gamma}\|v\|_{\Omega_{s}}\right\}, \quad v \in C^{\infty}\left(\Omega_{s}\right) .
$$

Choose any $\rho_{0}$ such that

$$
\gamma \leqq \mu \rho_{0} ;
$$

we prove the conclusion of the theorem for any $\rho \geqq \rho_{0}$.

Given an integer $k \geqq 1$ and a $\delta>0$, we apply (6) with $s=(1-1 / k) \delta$, $t=\delta / k$ and $v=Q^{(k-1) v}(D) u$. Next (if $k>1$ ) we apply again (6) to both terms on the right-hand side with $s=(1-2 / k) \delta, t=\delta / k$ and either $v=$ $Q^{(k-2) v}(D) P^{\mu}(D) u$ or $v=Q^{(k-2) v}(D) u$. After $k$ such steps we obtain

$$
\left\|Q^{k v}(D) u\right\|_{\Omega_{\delta}} \leqq C^{k} \sum_{i=0}^{k}\left(\begin{array}{c}
k \\
i
\end{array}\right)\left(\frac{k}{\delta}\right)^{i \gamma}\left\|P^{(k-i) \mu}(D) u\right\|_{\Omega}, \quad u \in C^{\infty}(\Omega) .
$$

Note that inequality (8) holds also (trivially) for $k=0$.

On the other hand, for $i=0,1, \cdots, v-1$, we have $\left|Q^{i}(\xi)\right|^{2} \leqq 1+\left|Q^{v}(\xi)\right|^{2}$, $\xi \in R^{n}$. Therefore, by the same theorem of Hörmander, there is a constant $C^{\prime}$ such that

$$
\left\|Q^{i}(D) v\right\|_{\Omega_{2 \delta}} \leqq C^{\prime}\left\{\left\|Q^{v}(D) v\right\|_{\Omega_{\delta}}+\|v\|_{\Omega_{\delta}}\right\}, \quad v \in C^{\infty}\left(\Omega_{\delta}\right),
$$

$i=0,1, \cdots, v-1$; here the constant $C^{\prime}$ depends on $\delta$.

If $j$ is an arbitrary integer $\geqq 0$, we can write

$$
j=k v+l \text {, }
$$

where $k, l$ are integers $\geqq 0$ and $l \leqq \nu-1$. Now we utilize (9) with $i=l$ and $v=Q^{k v}(D) u$ to see that

$$
\left\|Q^{j}(D) u\right\|_{\Omega_{2 \delta}} \leqq C^{\prime}\left\{\left\|Q^{(k+1) v}(D) u\right\|_{\Omega_{\delta}}+\left\|Q^{k v}(D) u\right\|_{\Omega_{\delta}}\right\}, \quad u \in C^{\infty}(\Omega) .
$$

This together with (8) implies that

$$
\begin{aligned}
\left\|Q^{j}(D) u\right\|_{\Omega_{2 \delta}} \leqq & C^{\prime} C^{k+1} \sum_{i=0}^{k+1}\left(\begin{array}{c}
k+1 \\
i
\end{array}\right)\left(\frac{k+1}{\delta}\right)^{i \gamma}\left\|P^{(k+1-i) \mu}(D) u\right\|_{\Omega} \\
& +C^{\prime} C^{k} \sum_{i=0}^{k}\left(\begin{array}{c}
k \\
i
\end{array}\right)\left(\begin{array}{l}
k \\
\delta
\end{array}\right)^{i \gamma}\left\|P^{(k-i) \mu}(D) u\right\|_{\Omega} .
\end{aligned}
$$

\footnotetext{
${ }^{1}$ In Hörmander's theorem $t$ is $\frac{1}{2}$ of the diameter of $\Omega$, but from the proof it is clear that $t$ can be any number $>0$.
} 
Suppose now that $u \in \Gamma_{P}^{\rho}\left(\Omega^{\prime}\right)$, where $\Omega^{\prime}$ is an arbitrary open set in $R^{n}$. If $K$ is a compact subset of $\Omega^{\prime}$, then there is a bounded open set $\Omega$ and a $\delta>0$ such that $K \subset \Omega_{2 \delta} \subset \Omega \subset \subset \Omega^{\prime}$. Consequently, for some constant $B$, $\left\|P^{i}(D) u\right\|_{\Omega} \leqq B^{i+1} i^{i \rho}, i=0,1, \cdots$, whence, in view of (7),

$$
k^{i \gamma}\left\|P^{(k-i) \mu}(D) u\right\|_{\Omega} \leqq\left(\mu^{\rho} B+1\right)^{k \mu+1} k^{k \mu \rho}, \quad i=0,1, \cdots, k .
$$

Applying (12) and (10) to the right-hand side of (11), we can find two constants $B_{1}$ and $B_{2}$ such that $\left\|Q^{j}(D) u\right\|_{K} \leqq\left\|Q^{j}(D) u\right\|_{\Omega_{2 \delta}} \leqq B_{1}^{k+1} k^{k \mu \rho} \leqq$ $B_{2}^{j+1} j^{j \rho \mu / v}=B_{2}^{j+1} j^{j \rho h}$. Thus $u \in \Gamma_{Q}^{\rho h}\left(\Omega^{\prime}\right)$, which proves the theorem.

In what follows we use Banach spaces formed of functions from $\Gamma_{P}^{\rho}(\Omega)$ or $\Gamma_{Q}^{\rho}(\Omega)$. Given any $N>0$, we denote by $\Gamma_{P}^{\rho . N}(\Omega)$ the linear space of functions $u \in \Gamma_{P}^{\rho}(\Omega)$ such that

$$
p_{\Omega, \rho, N}(u)=\sum_{k=0}^{\infty}(k !)^{-\rho} N^{-k-1}\left\|P^{k}(D) u\right\|_{\Omega}<\infty .
$$

It is easy to see that $\Gamma_{P}^{\rho . N}(\Omega)$, with $p_{\Omega, \rho, N}$ as the norm, is a Banach space. $\Gamma_{Q}^{\rho, N}(\Omega)$ is defined analogously and the corresponding norm is denoted by $q_{\Omega, \rho, N}$.

LEMMA Assume that

$$
\Gamma_{P}^{\rho}(\Omega) \subset \Gamma_{Q}^{\rho^{\prime}}(\Omega)
$$

for some $\rho, \rho^{\prime}>0$. Then, for every open set $\Omega^{\prime} \subset \subset \Omega$, there exists an $N>0$ such that

$$
\left\{u \mid \Omega^{\prime}: u \in \Gamma_{P}^{\rho, 1}(\Omega)\right\} \subset \Gamma_{Q}^{\rho^{\prime}, N}\left(\Omega^{\prime}\right) .
$$

Proof. Suppose that the lemma is false. Then there exists an open set $\Omega^{\prime} \subset \subset \Omega$ such that, for every $N>0$, one can find a function in $\Gamma_{P}^{\rho .1}(\Omega) \subset$ $\Gamma_{P}^{\rho}(\Omega) \subset \Gamma_{Q}^{\rho^{\prime}}(\Omega)$ whose restriction to $\Omega^{\prime}$ does not belong to $\Gamma_{Q}^{\rho^{\prime}, N}\left(\Omega^{\prime}\right)$. Thus, for $i=1,2, \cdots$, there exist positive numbers $A_{i}, B_{i}$, positive integers $k_{i}$, and functions $v_{i} \in \Gamma_{P}^{\rho, 1}(\Omega)$ with $p_{\Omega, \rho, 1}\left(v_{i}\right) \leqq 1$ such that

$$
\begin{gathered}
k_{i}<k_{i+1}, \\
4 B_{i}<A_{i+1}<B_{i+1}, \\
\left\|Q^{j}(D) v_{i}\right\|_{\Omega^{\prime}} \leqq B_{i}^{j+1}(j !)^{\rho^{\prime}}, \quad j=0,1, \cdots, \\
\left\|Q^{k_{i}}(D) v_{j}\right\|_{\Omega^{\prime}} \leqq\left(A_{i} / 4\right)^{k_{i}+1}\left(k_{i} !\right)^{\rho^{\prime}} \quad \text { for } j>i, \\
\left\|Q^{k_{i}}(D) v_{i}\right\|_{\Omega^{\prime}}>A_{i}^{k_{i}+1}\left(k_{i} !\right)^{\rho^{\prime}} .
\end{gathered}
$$

The function $v=\sum_{j=0}^{\infty} 2^{-j} v_{j}$ is in $\Gamma_{P}^{\rho \cdot 1}(\Omega)$ and therefore in $\Gamma_{P}^{\rho}(\Omega)$. But, in 
view of (15)-(19),

$$
\begin{aligned}
\left\|Q^{k_{i}}(D) v\right\|_{\Omega^{\prime}} & >2^{-i}\left\|Q^{k_{i}}(D) v_{i}\right\|_{\Omega^{\prime}}-\sum_{j \neq i} 2^{-j}\left\|Q^{k_{i}}(D) v_{j}\right\|_{\Omega^{\prime}} \\
& >\left(1 / 2^{i}-2 / 4^{k_{i}+1}\right) A_{i}^{k_{i}+1}\left(k_{i} !\right)^{\rho^{\prime}} \geqq\left(A_{i} / 2\right)^{k_{i}+1}\left(k_{i} !\right)^{\rho^{\prime}} .
\end{aligned}
$$

This means that $v$ does not belong to $\Gamma_{Q}^{\rho^{\prime}}(\Omega)$, which is a contradiction to (13). The lemma is thus proved.

THEOREM 2. If the inclusion (4) holds for some nonempty bounded open set $\Omega$ and some $\rho, h>0$, then the polynomials $P$ and $Q$ satisfy condition (3).

Proof. Let $\Omega^{\prime}$ be any nonempty open set such that $\Omega^{\prime} \subset \subset \Omega$. Then, by our hypothesis and the lemma (with $\rho^{\prime}=\rho h$ ), there exists an $N>0$ such that $\left\{u \mid \Omega^{\prime}: u \in \Gamma_{P}^{\rho, 1}(\Omega)\right\} \subset \Gamma_{Q}^{\rho h, N}\left(\Omega^{\prime}\right)$. Moreover, the restriction mapping $u \rightarrow u \mid \Omega^{\prime}$ of $\Gamma_{P}^{\rho, 1}(\Omega)$ into $\Gamma_{Q}^{\rho h, N}\left(\Omega^{\prime}\right)$ is closed and therefore continuous. Thus there is a constant $C$ such that $q_{\Omega^{\prime}, \rho h, N}\left(u \mid \Omega^{\prime}\right) \leqq C p_{\Omega, \rho, 1}(u), u \in \Gamma_{P}^{\rho, 1}(\Omega)$. In particular, for $u(x)=e^{i\langle\xi, x\rangle}, \xi \in R^{n}$, we obtain

$$
E_{\rho h}\left[\left(N^{-1}|Q(\xi)|\right)^{1 / \rho h}\right] \leqq C_{1} E_{\rho}\left(|P(\xi)|^{1 / \rho}\right), \quad \xi \in R^{n},
$$

with the function $E_{\lambda}(t), \lambda>0, t \geqq 0$, defined by $E_{\lambda}(t)=\sum_{j=0}^{\infty}\left(t^{j} / j !\right)^{\lambda}$ and another constant $C_{1}$.

But, for a fixed $\lambda$, there are $M, a>0$ such that

$$
e^{a t} \leqq M E_{\lambda}(t), \quad t \geqq 0,
$$

(see [4, Lemma 7.4]). On the other hand, since $(2 t)^{j} / j !<e^{2 t}$, for $t \geqq 0$, $j=0,1, \cdots$, we have

$$
E_{\lambda}(t)<e^{2 \lambda t} \sum_{j=0}^{\infty} 2^{-j \lambda}=\frac{e^{2 \lambda t}}{\left(1-2^{-\lambda}\right)}, \quad t \geqq 0 .
$$

We now use (21) and (22) to estimate both sides of inequality (20). This leads to the condition

$$
\exp \left(b_{1}|Q(\xi)|^{1 / \rho h}\right) \leqq C_{2} \exp \left(b_{2}|P(\xi)|^{1 / \rho}\right), \quad \xi \in R^{n}
$$

where $b_{1}, b_{2}, C_{2}$ are positive constants. Hence it follows that $|Q(\xi)|^{1 / \rho h} \leqq$ $C_{3}\left(1+|P(\xi)|^{1 / \rho}\right), \xi \in R^{n}$, with another constant $C_{3}$, and we see that condition (3) is satisfied. The proof is thus complete.

We now combine the above results with the following strengthened version of Komatsu's theorem, mentioned in the introduction.

THEOREM 3. If a polynomial $Q$ of order $m$ is elliptic then, for any open set $\Omega \subset R^{n}$ and any $\rho>1, \Gamma_{Q}^{\rho m}(\Omega) \subset \Gamma^{\rho}(\Omega)$.

Proof. Since $Q$ is elliptic, there is a constant $C$ such that $\left|\xi^{\alpha}\right|^{2} \leqq$ $C\left(1+|Q(\xi)|^{2}\right), \xi \in R^{n}$, for every multi-index $\alpha$ with $|\alpha| \leqq m$. Making use 
of this condition one can prove Theorem 3 by modifying slightly the proof of Theorem 1. We omit the details.

Corollary. Let $P$ be a hypoelliptic polynomial such that, for some constants $C, h>0$,

$$
|\xi|^{2} \leqq C\left(1+|P(\xi)|^{2}\right)^{h}, \quad \xi \in R^{n} .
$$

Then, for any open set $\Omega \subset R^{n}$ and large $\rho$,

$$
\Gamma_{P}^{\rho}(\Omega) \subset \Gamma^{\rho h}(\Omega) \text {. }
$$

Conversely, if the inclusion (24) holds for some nonempty bounded open set $\Omega$ and some $\rho, h>0$, then condition (23) is fulfilled.

Proof. For the Laplace operator $\Delta$, the generating polynomial is $Q(\xi)=|\xi|^{2}, \xi \in R^{n}$. Thus, by Theorem 1 and Theorem 3, condition (23) implies that, for any open set $\Omega$ and large $\rho, \Gamma_{P}^{\rho}(\Omega) \subset \Gamma_{Q}^{2 \rho h}(\Omega) \subset \Gamma^{\rho h}(\Omega)$.

Conversely, if (24) holds for some nonempty bounded open set $\Omega$ and some $\rho, h>0$, then $\Gamma_{P^{2}}^{\rho}(\Omega) \subset \Gamma^{2 \rho h}(\Omega) \subset \Gamma_{Q}^{\rho h}(\Omega)$. Therefore, by Theorem 2, condition (23) must be satisfied.

\section{REFERENCES}

1. L. Hörmander, Linear partial differential operators, 3rd ed., Die Grundlehren der math. Wissenschaften, Band 116, Springer-Verlag, New York, 1969. MR 40 \#1687.

2. - On interior regularity of the solutions of partial differential equations, Comm. Pure Appl. Math. 11 (1958), 197-218. MR 21 \#5064.

3. H. Komatsu, A characterisation of real analytic functions, Proc. Japan Acad. 36 (1960), 90-93. MR 24 \#A3425.

4. F. Trèves, Linear partial differential equations with constant coefficients: Existence, approximation and regularity of solutions, Math. Appl., vol. 6, Gordon and Breach, New York, 1969. MR 37 \#557.

Department of Mathematics, State University of New York, College at Buffalo, Buffalo, NeW York 14222

Department of Mathematics, State University of New York at Buffalo, AMHERST, NEW YoRK 14226 